\title{
Path Integral Quantization of Regular Lagrangian
}

\author{
Ola A. Jarab'ah ${ }^{1}$ \\ ${ }^{1}$ Applied Physics Department, Faculty of Science, Tafila Technical University, Jordan \\ Correspondence: Ola A. Jarab'ah, Applied Physics Department, Faculty of Science, Tafila Technical University, \\ Jordan. E-mail: oasj85@yahoo.com \\ Received: December 6, 2017 \\ Accepted: December 20, $2017 \quad$ Online Published: January 15, 2018 \\ doi:10.5539/apr.v10n1p9 \\ URL: https://doi.org/10.5539/apr.v10n1p9
}

\begin{abstract}
Path integral formulation based on the canonical method is discussed. The Hamilton Jacobi function for regular Lagrangian is obtained using separation of variables method. This function is used to quantize regular systems using path integral method. The path integral is obtained as integration over the canonical phase space coordinates. One illustrative example is considered to demonstrate the application of our formalism.
\end{abstract}

Keywords: Hamilton Jacobi Equation, Regular Systems, Path Integral, Quantization

\section{Introduction}

The path integral is an expression for the propagator in terms of an integral over an infinite dimensional space of paths in configuration space. Many important applications of path integral have been found in statistical physics, in the theory of phase transitions, super fluidity, super conductivity, quantum optics, and plasma physics.

The path integral concept was introduced for the first time by (Wiener, 1921) as a method to solve problems in the theory of diffusion and Brownian motion. This integral which is now also called the Wiener integral has played a central role in the further development of the subject of path integration.

It was reinvented in a different form by (Feynman, 1948), for the reformulation of quantum mechanics. The Feynman approach was inspired by Dirac's paper on the role of the Lagrangian and the least- action principle in quantum mechanics (Dirac, 1933).

The quantization of Lagrangian systems was discussed in most references of classical mechanics (Razavy, 2005). Moreover, the quantization of constrained systems has been studied using the WKB approximation by (Rabei et al., 2002, Rabei \& Guler 1992; Nawafleh, 2002, Nawafleh et al., 2004; Hasan et al., 2004). Recently the quantization of dissipative systems has been studied using the WKB approximation by (Jarab'ah et al., 2013). The path integral quantization of constrained Lagrangian systems has been investigated by (Muslih \& Guler, 1997; Rabei, 2000; Muslih, 2001, 2002); also the path integral quantization of dissipative systems has been investigated by (Hasan, 2014).

The purpose of the present work is to construct the Hamilton Jacobi function for regular Lagrangian using separation of variables technique in order to quantize the regular systems using path integral method.

This paper is organized as follows. In section 2, Hamilton Jacobi formulation and path integral quantization were discussed. In section 3, illustrative example is examined. In section 4, the work closes with some concluding remarks.

\section{Hamilton Jacobi Formulation and Path Integral Quantization}

The Lagrangian formulation of classical system requires that, the system is formulated by the generalized coordinates $q_{i}$ and velocities $\dot{q}_{i}$, according to that, we can write the Lagrangian as a function of these general coordinates (regular Lagrangian) $L_{\circ}=L_{\circ}(q, \dot{q}, t)$, and the corresponding Euler Lagrange equation is given by (Thornton, 2004).

$$
\frac{d}{d t}\left(\frac{\partial L_{\circ}}{\partial \dot{q}}\right)-\frac{\partial L_{\circ}}{\partial q}=0
$$

According to that, the canonical Hamiltonian can be written as 
where, the momentum can be obtained from

$$
H=p \dot{q}-L_{\circ}
$$

$$
p=\frac{\partial L_{\circ}}{\partial \dot{q}}
$$

As a result, the Hamiltonian is constructed in the system, and one can write the Hamilton Jacobi equation like

$$
H^{\prime}\left(q, \frac{\partial S}{\partial q}, \frac{\partial S}{\partial t}, t\right)=0
$$

where

$$
H^{\prime}=H\left(q, \frac{\partial S}{\partial q}\right)+\frac{\partial S}{\partial t}
$$

The momentum now is

$$
p=\frac{\partial S}{\partial q}
$$

It is known that in standard formulation the Hamilton Jacobi problem focuses on finding $S(q, t)$, which satisfies equation(4) and one can achieve that by using the method of separation of variables, by assuming $S(q, \alpha, t)=w(q, \alpha)-\alpha t$, (Goldstein,1980), where $\alpha$ is a constant, and $w(q, \alpha)$ is time independent and called Hamilton's characteristic function, it follows that

$$
H\left(q, \frac{\partial S}{\partial q}\right)=\alpha
$$

In the canonical method the action function and the equations of motion are written as total differential equations as follows

$$
\begin{gathered}
d q=\frac{\partial H^{\prime}}{\partial p} d t \\
d p=-\frac{\partial H^{\prime}}{\partial q} d t \\
d z=-\frac{\partial S}{\partial t} d t+\frac{\partial S}{\partial q} d q=\left(-H+p \frac{\partial H^{\prime}}{\partial p}\right) d t
\end{gathered}
$$

Where

$Z=S(q, t)$ is the Hamilton Jacobi function, which is obtained in terms of the canonical coordinates.

The path integral representation may be written as

$$
D(q, p)=\int \exp (i Z) d p d q=\int\left[\exp i\left\{\int\left(-H+p \frac{\partial H^{\prime}}{\partial p}\right) d t\right\}\right] d p d q
$$

\section{Illustrative Example}

Our formalism can be illustrated by discussing the following example:

Consider a particle of mass $\mathbf{m}$ moves vertically under the influence of gravity, without frictional forces (Jarab'ah, 2013). The Lagrangian is given by

$$
L_{\circ}=\frac{1}{2} m \dot{y}^{2}-m g y
$$

The canonical Hamiltonian is

$$
H=p \dot{y}-L_{\circ}
$$


and the canonical momentum can be calculated using

$$
P=\frac{\partial L_{\circ}}{\partial \dot{y}}=m \dot{y}
$$

Then,

$$
\dot{y}^{2}=\frac{p^{2}}{m^{2}}
$$

Thus, the canonical Hamiltonian can be written as the following form

$$
H=\frac{p^{2}}{2 m}+m g y
$$

The corresponding Hamiltonian Jacobi equation reads

$$
\frac{p^{2}}{2 m}+m g y+\frac{\partial S}{\partial t}=0
$$

The solution of the Hamiltonian Jacobi equation can be constructed as

$$
S(y, t)=y f(t)+R(t)
$$

Then,

$$
\frac{\partial S}{\partial y}=f(t)
$$

Using equation (6), the function $f(t)$ represents the canonical momentum, so that

$$
f(t)^{2}=p^{2}
$$

Taking the first time derivative of equation (18) this yields

$$
\frac{\partial S}{\partial t}=y f^{\prime}(t)+R^{\prime}(t)
$$

Inserting equation (20) and equation (21) into equation (17) we obtain

$$
\frac{1}{2 m} f^{2}(t)+m g y+y f^{\prime}(t)+R^{\prime}(t)=0
$$

Matching power of $y$, we get

$$
-m g y=y f^{\prime}(t)
$$

After integration

$$
f(t)=-m g t+f \text { 。 }
$$

Choose $f_{\circ}=0$, equation (24) becomes

$$
f(t)=-m g t
$$

Then,

$$
p^{2}=f^{2}(t)=(-m g t)^{2}
$$

and

$$
-\frac{1}{2 m} f^{2}(t)=R^{\prime}(t)
$$

Then, 


$$
-\frac{m g^{2} t^{3}}{6}+R_{\circ}=R(t)
$$

Similarly choose $R_{\circ}=0$, equation (28) becomes

$$
-\frac{m g^{2} t^{3}}{6}=R(t)
$$

Making use of equation (25) and (29), the Hamilton Jacobi function takes the following form

$$
S=-m g t y-\frac{1}{6} m g^{2} t^{3}
$$

Remembering that

$$
p=-m g t
$$

So that, equation (30) gives

$$
S=y p+\frac{1}{6} g t^{2} p
$$

Let $c=\frac{1}{6} g t^{2}$

The final form of the Hamiltonian function is

$$
Z=S=(y+c) p
$$

Now we come to the quantization of our system using path integral representation.

Using equation (11) the path integral for this example is

$$
D(y, p)=\iint\{\exp [i p(y+c)]\} d p d y
$$

The path integral representation can be written as

$$
D(y, \dot{y})=-i \ln |p|-i \sum_{n=1}^{\infty} \frac{[i p(y+c)]^{n}}{n . n !}+N(y)+D(p)
$$

\section{Conclusion}

The path integral formulation of regular systems was studied within the framework of Hamilton Jacobi equation. The Hamiltonian treatment of regular systems gives the Hamilton Jacobi equation, which leads to obtain the action function $\mathbf{S}$ using the technique of separation of variables. Then the path integral is obtained directly as an integration over the canonical phase space coordinates $q_{i}$ and $\dot{q}_{i}$. An illustrative example was examined.

\section{References}

Dirac, P. A. M. (1933). The Lagrangian in Quantum Mechanics. Physikalische Zeitschrift der Sowjetunion, 3, 64-72. https://doi.org/10.1142/9789812567635_0003/the Lagrangian in quantum mechanics

Feynman, R. P. (1948). Space-Time Approach to Non-Relativistic Quantum Mechanics. Reviews of Modern Physics, 20(2), 367-387. http://link.aps.org/doi/10.1103/RevModPhys.20.367

Goldstein, H. (1980). Classical Mechanics (2nd ed.). Reading-Massachusetts: Addison-Wesley.

Hasan, E. H. (2014). Path Integral Quantization of Lagrangian with Linear Accelerations. European Scientific Journal, 10(3), 331-345. http://eujournal.org/index.php/esj/article/viewfile/2632/2493

Hasan, E. H., Rabei, E. M., \& Ghassib, H. B. (2004). Quantization of Higher-Order Constrained Lagrangian Systems Using the WKB Approximation. International Journal of Theoretical Physics, 43(4), 1073-1096. Retrieved from http://link.springer.com/article/10.1023/B:IJTP.0000049027.45011.37

Jarab'ah, O., Nawafleh, K., \& Ghassib, H. (2013). A Hamilton-Jacobi Treatment of Dissipative Systems. European Scientific Journal, 9(30), 70-81. Retrieved from http://www.eujournal.org/index.php/esj/article/ download/1946/1888 
Jarab'ah, O., Nawafleh, K., \& Ghassib, H. (2013). Canonical Quantization of Dissipative Systems. European Scientific Journal, 9(36), 132-154. Retrieved from http://eujournal.org/index.php/esj/article/download/ $2218 / 2102$

Muslih S., \& Guler, Y. (1997). The Feynman Path Integral Quantization of Constrained Systems. Il Nuovo Cimento B, 112(1), 97-107. Retrieved from http://inis.iaea.org/search/search.aspx?orig_q=RN28069286

Muslih, S. (2001). Path Integral Formulation of Constrained Systems with Singular-Higher Order Lagrangians. Hadronic Journal, 24(6), 713-720. Retrieved from https:// arxiv.org.math.ph

Muslih, S. (2002). Quantization of Singular Systems with Second-Order Lagrangians. Modern Physics Letters A, 17(36), 2383-2391. Retrieved from http://www.worldscientific.com/doi /pdf/10.1142/S0217732302009027

Nawafleh, K. I. (2002). Quantization of Constrained Systems Using the WKB Approximation (PhD. thesis, University of Jordan, Amman, Jordan).

Nawafleh, K. I., Rabei, E. M., \& Ghassib, H. B. (2004). Hamilton -Jacobi Treatment of Constrained Systems. International Journal of Modern PhysicsA, 19 (3), 347-354. Retrieved from https://www.worldscientific.com/ doi/pdf/10.1142/S0217751X04017719

Rabei, E. (2000). On the Quantization of Constrained Systems Using Path Integral Techniques. Il Nuovo Cimento B, 115(10), 1159-1165. Retrieved from http:// adsabs.harvard.edu/abs/2000NCimB.115.1159R

Rabei, E., \& Guler, Y. (1992). Hamilton-Jacobi Treatment of Second-Class Constraints. Physical Review A, 46(6), 3513-3515. Retrieved from http://link.aps.org/pdf/101103/physRevA.46.3513

Rabei, E., Nawafleh, K., \& Ghassib, H. (2002). Quantization of Constrained Systems Using the WKB Approximation. Physical Review A, 66(2) 024101. Retrieved from http://link.aps.org/10.1103/PhysRevA. 66.024101

Razavy, M. (2005). Classical and Quantum Dissipative Systems. London Imperial College Press.

Thornton, T., \& B. Marion. (2004). Classical Dynamics of Particles and Systems (5th ed.). Library of Congress, United States of America.

Wiener, N. (1921). The Average of an Analytical Functional and the Brownian Movement. Proceedings of the National Academy of Sciences of the United States of America, 7(10), 294-298.

\section{Copyrights}

Copyright for this article is retained by the author(s), with first publication rights granted to the journal.

This is an open-access article distributed under the terms and conditions of the Creative Commons Attribution license (http://creativecommons.org/licenses/by/4.0/). 